\title{
Avaliação do estado nutricional de idosos utilizando o índice de massa corporal e a mini avaliação nutricional
}

\author{
Jozélia Soares Bueno*; Leucinéia Schmidt"; Rúbia Garcia Deon"*;; Dionara Simoni Hermes Volkweis"*;;
}

\section{Resumo}

Os idosos podem apresentar alterações no estado nutricional. As alterações mais importantes na composição corporal são o aumento da gordura corporal total e a redução do tecido muscular, que contribuem desta forma, para a ocorrência de distúrbios nutricionais, como a desnutrição ou o excesso de peso. Este estudo objetivou avaliar o estado nutricional de idosos frequentadores de um grupo de convivência no município de Boa Vista das Missões/RS. Participaram 16 idosos, sendo que a maioria $(81,3 \%)$ eram mulheres e tinham idade entre 61 e 79 anos. A coleta de dados se deu a partir do índice de massa corporal e da mini avaliação nutricional, além de um questionário com perguntas fechadas para a caracterização da amostra. Quanto ao estado civil, a maioria (62,5\%) eram casados ou viviam com companheiros e $87,5 \%$ residiam com seus familiares. $O$ índice de massa corporal apontou que 56,3\% apresentava excesso de peso; já os resultados da mini avaliação nutricional demostraram que $50 \%$ apresentaou risco de desnutrição. A maioria dos idosos apresentaram excesso de peso quando avaliados pelo índice de massa corporal e risco de desnutrição quando avaliados pela mini avaliação nutricional. Nas idades mais avançadas verificou-se que a prevalência de excesso de peso diminuiu quando avaliado por meio do índice de massa corporal e o risco para desnutrição aumentou quando avaliado através da mini avaliação nutricional.

Palavras-chave: Idoso. Índice de massa corporal. Mini avaliação nutricional.

\section{Introdução}

De acordo com os dados do Instituto Brasileiro de Geografia e Estatística (IBGE), de 2015 para 2016, a expectativa de vida do brasileiro ao nascer passou de 75,5 para 75,8 anos, o que representa um acréscimo de três meses e onze dias. De 1940 até 2016, o aumento foi de 30,3 anos. Apesar do crescimento contínuo na expectativa de vida, o Brasil ainda está abaixo de países como Japão, Itália, Singapura e Suíça, que em 2015 tinham

* Nutricionista pela Universidade Regional Integrada do Alto Uruguai e das Missões Campus de Frederico Westphalen. E-mail: jozysbueno@hotmail.com.

*** Nutricionista. Especialista em Saúde do Idoso pela Universidade de Passo Fund. E-mail: leucineia@hotmail. com.

*** Nutricionista. Doutora em Gerontologia Biomédica pela Pontifícia Universidade Católica do Rio Grande do Sul. Mestre em Saúde Coletiva pela Universidade Luterana do Brasil. E-mail: rubiadeon@yahoo.com.br..

**** Nutricionista. Pós-Graduada em Saúde Pública pela Universidade Regional do Noroeste do Estado do Rio Grande do Sul. Mestrado em Envelhecimento Humano pela Universidade de Passo Fundo. E-mail: dshermes@uri.edu.br.

$\rightarrow$ http://dx.doi.org/10.5335/rbceh.v16i3.8372 
o indicador na faixa dos 83 anos (BRASIL, 2017).

$\mathrm{O}$ estado nutricional assume uma importante função na qualidade de vida e saúde dos idosos. Por um lado, o excesso de peso consolidou-se como agravo nutricional associado à alta incidência de Doenças Crônicas Não Transmissíveis (DCNTs), tais como doenças cardiovasculares, câncer e diabetes, influenciando, desta maneira, no perfil de morbimortalidade das populações (TOMASI et al., 2014; GARCIA; MORETTO; GUARIENTO, 2016). Por outro lado, a desnutrição apresenta-se fortemente associada ao aumento da incapacidade funcional, aumento no número de internações, redução da qualidade de vida, maior susceptibilidade às infecções e, consequentemente, aumento da mortalidade (ALMEIDA et al., 2013; SOUZA et al., 2014).

Com o envelhecimento as pessoas ficam menos ativas, reduzindo a capacidade física, bem como, o bom funcionamento de seus órgãos e sistemas. Estes fatores facilitam o aparecimento das DCNTs, podendo estas ser determinantes no estado nutricional, prejudicando o processo de envelhecimento (ROCHA et al., 2016). O índice de massa corporal (IMC) e da mini avaliação nutricional (MNA) são dois instrumentos muito eficazes na avaliação do estado nutricional dos idosos, não invasivos, de fácil aferição e com baixo custo. O IMC é o indicador antropométrico mais utilizado para avaliação do estado nutricional, por ser uma medida de fácil aplicabilidade e pequena variação entre os avaliadores
(CORTEZ; MARTINS, 2012). Lipschitz (1994) propôs uma classificação para o IMC que considera as modificações corporais que ocorrem no envelhecimento e a vulnerabilidade dos idosos à desnutrição. A MNA também constitui um método simples e rápido de ser aplicado, estabelecendo boa correlação entre morbidade e mortalidade, podendo ser utilizada para identificar indivíduos que estão no estado nutricional normal, em risco de desnutrição ou desnutridos (MUSSOI, 2014).

Desta forma, este estudo teve como objetivo principal a avaliação do estado nutricional dos idosos frequentadores de um grupo de convivência no município de Boa Vista das Missões/RS.

\section{Materiais e métodos}

Trata-se de um estudo transversal, quantitativo e descritivo realizado com 16 idosos de ambos os sexos. A seleção dos participantes foi realizada através da lista de cadastro dos idosos frequentadores de um grupo de Convivência no município de Boa Vista das Missões/RS. Foram incluídos nesta pesquisa todos os indivíduos acima de 60 anos que aceitaram participar voluntariamente da pesquisa. A coleta de dados foi realizada individualmente, na própria instituição. A avaliação do estado nutricional se deu partir do IMC e da MNA. Além disso, foi aplicado um questionário com perguntas fechadas para a caracterização da amostra sobre o sexo, idade, estado civil, se residiam com familiares ou sozinhos. 
O peso corporal foi mensurado em balança digital da marca TianShan ${ }^{\circledR}$ (com capacidade para $150 \mathrm{Kg}$ ), com os idosos vestindo roupas leves, descalços, em posição ereta, com pernas e calcanhares juntos e braços ao longo do corpo. A aferição da estatura foi realizada com auxílio de antropômetro vertical fixo à balança (capacidade para $200 \mathrm{~cm}$ ) com os idosos em pé, descalços, em posição ortostática, com os calcanhares juntos, costas retas e braços estendidos ao longo do corpo (CUPPARI et al., 2005). O cálculo do IMC foi realizado através do peso (em quilos) dividido pelo quadrado da altura (em metros). Para a classificação do IMC utilizou-se os pontos de corte sugeridos por Lipschitz (1994), que levam em consideração as modificações corporais que ocorrem durante o processo de envelhecimento.

A MNA compreende 18 perguntas agrupadas em 4 categorias: avaliação antropométrica; avaliação geral do estilo de vida, uso de medicamentos, mobilidade; avaliação dietética e a autoavaliação (percepção da saúde). O escore total obtido por meio da soma dos pontos, acima de 23,5, indica bom estado nutricional, de 17 a 23,5, risco de desnutrição e abaixo de 17 , desnutrição. Na avaliação antropométrica, avalia-se peso, altura, circunferência do braço e circunferência da panturrilha (GUIGOZ; VELLAS; GARRY, 1994). Os resultados desta pesquisa foram expressos em tabelas em forma de percentual. A significância estatística foi definida como $\mathrm{p}<0,05$. Os dados foram tabulados e analisados com auxílio do programa SPSS 17.0.
Os testes utilizados para as associações entre os dados foram o Qui-quadrado e a correlação linear.

Todos os participantes realizaram a assinatura do Termo de Consentimento Livre e Esclarecido (TCLE). O estudo foi aprovado pelo Comitê de Ética em Pesquisa (CEP) da Universidade Regional do Alto Uruguai e das Missões (URI), campus de Frederico Westphalen/RS, sob número CAAE: 25008813.4.0000.5352.

\section{Resultados}

Participaram da pesquisa 16 idosos, sendo que $13(81,2 \%)$ eram mulheres. Quanto a idade, $3(18,8 \%)$ tinham entre 60 e 64 anos, $3(18,8 \%)$ entre 65 e 69 anos, $6(37,5 \%)$ entre 70 e 74 anos e 4 (25\%) entre 75 e 79 anos. Com relação ao estado civil, $10(62,5 \%)$ eram casados ou viviam com companheiro, 5 (31,3\%) eram viúvos e $1(6,3 \%)$ era separado ou divorciado. Entre os frequentadores do grupo, $14(87,5 \%)$ moravam com seus familiares e $2(12,5 \%)$ moravam sozinhos. Conforme demonstra a Tabela 1 , quanto ao IMC, $2(12,5 \%)$ apresentavam baixo peso, $5(31,2 \%)$ apresentavam eutrofia e $9(56,3 \%)$ estavam com excesso de peso. 
Tabela 1. Associação entre sexo e IMC.

\begin{tabular}{|l|l|l|l|}
\hline \multirow{2}{*}{ IMC } & \multicolumn{2}{c|}{ Sexo } & \multirow{2}{*}{ Total } \\
\cline { 2 - 3 } & \multicolumn{1}{|c|}{ Masculino } & \multicolumn{1}{c|}{ Feminino } & \\
\hline Baixo peso & $0(0,0)$ & $2(15,4)$ & $2(12,5)$ \\
\hline Eutrofia & $2(66,7)$ & $3(23,1)$ & $5(31,3)$ \\
\hline Excesso de peso & $1(33,3)$ & $8(61,5)$ & $9(56,3)$ \\
\hline
\end{tabular}

A associa entre sexo e IMC não foi significativa $(\mathrm{p}=0,318)$. Verificou-se que a maioria $(66,7 \%)$ dos homens apresentavam eutrofia e a maioria $(61,5 \%)$ das mulheres estavam com excesso de peso. A Tabela 2 apresenta a associação entre idade e IMC.

Tabela 2. Correlação entre idade e IMC.

\begin{tabular}{|l|l|l|l|l|l|}
\hline \multirow{2}{*}{ IMC } & \multicolumn{5}{c|}{ Idade } \\
\cline { 2 - 6 } & \multicolumn{1}{|c|}{$60-64$ anos } & $65-69$ anos & $\mathbf{7 0 - 7 4}$ anos & 75-79 anos & \multicolumn{1}{c|}{ Total } \\
\hline Baixo peso & $2(66,7)$ & $0(0,0)$ & $0(0,0)$ & $0(0,0)$ & $2(12,5)$ \\
\hline Eutrofia & $0(0,0)$ & $0(0,0)$ & $3(50,0)$ & $2(50,0)$ & $5(31,3)$ \\
\hline Excesso de peso & $1(33,3)$ & $3(100)$ & $3(50,0)$ & $2(50,0)$ & $9(56,3)$ \\
\hline
\end{tabular}

Os resultados apresentados na Tabela 2 apontam associação significativa entre idade e IMC $(p=0,042)$. A Tabela 2 indica que $100 \%$ dos idosos nas idades entre 65 e 69 anos apresentavam excesso

Tabela 3. Associação entre sexo e MNA.

\begin{tabular}{|l|l|l|l|}
\hline \multirow{2}{*}{ MNA } & \multicolumn{2}{c|}{ Sexo } & \multirow{2}{*}{ Total } \\
\cline { 2 - 3 } & \multicolumn{1}{|c|}{ Masculino } & \multicolumn{1}{c|}{ Feminino } & \\
\hline Eutrofia & $2(66,7)$ & $5(38,0)$ & $7(44,0)$ \\
\hline Risco de desnutrição & $1(33,3)$ & $7(54,0)$ & $8(50,0)$ \\
\hline Desnutrido & $0(0,0)$ & $1(8,0)$ & $1(6,0)$ \\
\hline
\end{tabular}

Os dados apresentados na Tabela 3 indicam que não houve associação significativa entre sexo e a MNA $(p=0,644)$. Os homes apresentaram maior prevalência de eutrofia e as mulheres maior de peso e que nas idades mais avançadas esse percentual diminuiu. A Tabela 3 demonstra que a associação entre sexo e a MNA. 
Tabela 4. Associação entre idade e MNA.

\begin{tabular}{|l|l|l|l|l|l|}
\hline \multirow{2}{*}{ MNA } & \multicolumn{5}{|c|}{ Idade } \\
\cline { 2 - 6 } & \multicolumn{1}{|c|}{$\mathbf{6 0 - 6 4}$ anos } & $\mathbf{6 5 - 6 9}$ anos & $\mathbf{7 0 - 7 4}$ anos & $\mathbf{7 5 - 7 9}$ anos & \multicolumn{1}{c|}{ Total } \\
\hline Eutrofia & $1(33,3)$ & $1(33,3)$ & $3(50,0)$ & $2(50,0)$ & $7(44,0)$ \\
\hline Risco de desnutrição & $1(33,3)$ & $2(66,7)$ & $3(50,0)$ & $2(50,0)$ & $8(50,0)$ \\
\hline Desnutrição & $1(33,3)$ & $0(0,0)$ & $0(0,0)$ & $0(0,0)$ & $1(6,0)$ \\
\hline
\end{tabular}

Os resultados descritos na Tabela 4 indicam que ocorreu maior risco de desnutrição nos idosos com o aumento da idade. No entanto, a associação entre idade e MNA não foi significativa ( $\mathrm{p}=$ $0,556)$. A Tabela 5 apresenta a associação entre IMC e MNA.

Tabela 5. Associação entre IMC e MNA.

\begin{tabular}{|l|l|l|l|l|}
\hline \multirow{2}{*}{ IMC (\%) } & \multicolumn{4}{c|}{ Idade } \\
\cline { 2 - 5 } & \multicolumn{1}{|c|}{ Eutrófico } & \multicolumn{1}{c|}{$\begin{array}{c}\text { Risco de } \\
\text { desnutrição }\end{array}$} & \multicolumn{1}{c|}{ Desnutrição } & Total \\
\hline Abaixo do peso & $0(0,0)$ & $1(12,5)$ & $1(100)$ & $2(12,5)$ \\
\hline Eutrófico & $3(42,9)$ & $2(25,0)$ & $0(0,0)$ & $5(31,3)$ \\
\hline Sobrepeso/Obesidade & $4(57,1)$ & $5(62,5)$ & $0(0,0)$ & $9(56,3)$ \\
\hline
\end{tabular}

Os resultados apresentados na Tabela 5 indicam que não houve associação significativa $(p=0,080)$ entre IMC e MNA.

\section{Discussão}

No presente estudo verificou-se que a maioria $(81,2 \%)$ dos idosos era do sexo feminino. Santos et al. (2017) no estudo que realizaram observaram que do total de idosos, $80,60 \%$ eram do sexo feminino. Também, Pereira et al. (2018) verificaram prevalência de $73,02 \%$ de mulheres. Estes dados são compatíveis com a realidade da distribuição da população brasileira acima de 60 anos, que é constituída por $55,53 \%$ de idosos do sexo feminino (BRASIL, 2010).
De acordo com o IMC, neste estudo ocorreu maior prevalência $(56,3 \%)$ de excesso de peso. Zattar et al. (2013) encontraram $52,6 \%$ dos idosos de Florianópolis com excesso de peso. Salmaso et al. (2014) também verificaram excesso de peso em $54 \%$ dos idosos atendidos no ambulatório do Hospital Universitário Clementino Fraga Filho da Universidade Federal do Rio de Janeiro. Estudos internacionais também têm comprovado a elevada prevalência de excesso de peso na população idosa, em contraste ao baixo peso, fenômeno mundialmente conhecido como transição nutricional (BEFORT; NAZIR; PERRI, 2012; HABIB, 2013).

Neste estudo, conforme o IMC, o excesso de peso foi mais prevalente no sexo feminino $(61,5 \%)$. Outro estudo também verificou maior percentual de sobrepeso 
nas mulheres (62,5\%) (SCHERER et al., 2013). Ainda, Pereira, Spyrides e Andrade (2016) evidenciaram que o sobrepeso é mais prevalente entre as idosas.

$\mathrm{O}$ índice elevado de excesso de peso entre as mulheres pode estar relacionado ao maior acúmulo de gordura abdominal e à maior expectativa de vida, o que se torna preocupante pela associação da obesidade com várias DCNTs, aumentando com isso a morbimortalidade e diminuindo a qualidade de vida (SCHERER et al., 2013).

Com base no IMC, verificou-se neste estudo que nas idades mais avançadas ocorreu uma redução dos índices de excesso de peso. Menezes et al. (2013) em um estudo de base populacional realizado em Campina Grande, também relataram menores prevalências de sobrepeso quanto maior a faixa etária dos idosos. Ainda, Volpini e Frangella (2013) observaram tendência à diminuição do IMC nas faixas etárias mais elevadas. A diminuição do IMC com o avançar da idade pode ocorrer pela redução da massa muscular e devido à diminuição da quantidade de gordura corporal (SOUZA et al., 2013).

No presente estudo, através da MNA verificou-se $50 \%$ de risco para desnutrição e 6\% de desnutrição entre os idosos. Sperotto e Spinelli (2010) demostraram por meio da MNA, que $65 \%$ dos idosos estavam com risco de desnutrição e 35\% estavam desnutridos. Também, outro estudo identificou através a MNA, que a maioria $(69,2 \%)$ dos idosos institucionalizados da capital paulista tinha risco de desnutrição e 15,4\% eram desnutridos
(MANTOVANI; VIEBIG; MORIMOTO, 2018).

A MNA é considerada como o melhor método de triagem e avaliação nutricional em idosos. A natureza qualitativa e quantitativa da MNA permite o acompanhamento das mudanças nutricionais, sendo um instrumento prático e rápido para avaliar o risco nutricional dos idosos. Ela é uma ferramenta sensível o suficiente para detectar mínimas mudanças no estado nutricional que podem ocorrer ao longo do tempo. Sempre que possível, o nutricionista deve completar a avaliação com inquéritos dietéticos, que proporcionam melhor compreensão dos déficits de nutrientes (SILVA et al., 2015; CAVALCANTE; COUTINHO; BURGOS, 2017).

Na correlação entre IMC e MNA não houve diferenças significativas. Cardozo et al. (2017) verificaram que a maioria $(54,6 \%)$ dos idosos apresentava sobrepeso segundo o IMC, e de acordo com a MNA a maioria $(63,0 \%)$ apresentava eutrofia. Estudos demonstram que isso ocorre em decorrência da provável sensibilidade e especificidade da MNA para detecção precoce do risco nutricional (GUEDES; GAMA; TIUSSI, 2008; GUIGOZ; VELLAS; GARRY, 1994).

Paz, Fazzio e Santos (2012) avaliaram idosos institucionalizados utilizando o IMC e a MNA. Conforme o IMC, os idosos foram classificados com desnutrição $(37,5 \%)$, eutrofia $(37,5 \%)$ e sobrepeso (25\%). E de acordo com a MNA, a prevalência de eutrofia foi de $66,7 \%$, seguida por $25 \%$ de risco de desnutrição e $8,3 \%$ de desnutrição. 
Pereira et al. (2015) estudaram idosos residentes de 15 ILPI na cidade de Salvador e concluíram que, tanto pelo IMC quanto pela MNA, a maioria dos participantes estavam desnutridos e em risco de desnutrição. Santana et al. (2016), no estudo que realizaram verificaram através da MNA, que 76,2\% dos idosos apresentaram risco de desnutrição, e 14,3\% eram desnutridos. $O$ cuidado nutricional deve ser planejado de acordo com a avaliação nutricional, e intervenções devem ser adaptadas e desenvolvidas para cada situação (ONGAN; RAKICIOGLU, 2015).

Alencar, Leitão e Prado (2015), verificaram que $18,8 \%$ dos indivíduos hospitalizados que eram eutróficos, evoluíram para desnutrição. Hernández $\square$ Luis et al. (2018), em um estudo realizado com 298 pacientes idosos hospitalizados, encontraram uma alta prevalência de desnutrição, sendo que a mortalidade em 100 dias foi de 15,1\%. Outro estudo realizado no México, analisando dados referentes ao diagnóstico na admissão de pacientes com desnutrição prévia, conclui que é um fator de risco para o desenvolvimento de complicações e mortalidade, aumentando o risco para 2.64 vezes (PEREZ-FLORES et al., 2016).

A desnutrição e os distúrbios nutricionais estão diretamente associados a perdas funcionais, o que dificulta a recuperação em pacientes hospitalizados. Além disso, a perda de peso involuntária em idosos tem por consequência a maior permanência hospitalar e mortalidade (SANCHEZ-RODRIGUEZ et al., 2017).
A desnutrição é um problema comum na velhice, sendo considerado o distúrbio mais importante nesta fase da vida, que pode contribuir para o aumento da mortalidade, aumento da susceptibilidade às infecções e redução da qualidade de vida dos idosos. Apresenta causas multifatoriais, e está associada a problemas de saúde comumente observados, como as DCNTs, deficiências físicas e cognitivas, depressão, problemas de mastigação, dificuldades para deglutição e dependência para se alimentar (SOUSA et al., 2014).

O idoso apresenta diversas peculiaridades quanto ao consumo de alimentos, e no processo de envelhecimento ocorrem modificações sensoriais e fisiológicas, que, em muitos casos, prejudicam a ingestão adequada de nutrientes (MARTINS et al., 2016). As alterações sensoriais diminuem a sensibilidade por gostos primários (doce, amargo, salgado, ácido). Além disso, ocorrem diminuições na acuidade visual, na audição e no olfato, que são fatores muito relevantes na diminuição do consumo alimentar (MONTEIRO, 2009).

A redução do apetite, a perda de peso involuntária e a caquexia são comuns na população idosa, assim como a deficiência nutricional, todavia, não devem ser associadas ao processo normal de envelhecimento, podendo aumentar o risco de morbidade e mortalidade entre os idosos (SANTOS; MACHADO; LEITE, 2010).

$O$ contexto de vida em que se insere a maioria dos idosos brasileiros, sujeitos aos efeitos cumulativos acarretados pelo baixo nível econômico, baixa escolaridade e ausência de suporte social, exige 
a incorporação de uma abordagem de cuidado ampliado. A alimentação tem papel importante neste processo, principalmente, na etiologia de doenças associadas à idade, ao declínio funcional e ao surgimento de deficiências (INZITARI et al., 2011).

A utilização de mais de um método de triagem nutricional é importante para se obter maior precisão na avaliação, pois nem todo paciente com sobrepeso pode ser considerado nutrido (AZEVEDO et al., 2016). Avaliações do estado nutricional, do comportamento alimentar e das condições sociais em que os idosos estão inseridos, considerando a complexa rede de determinantes diretos e indiretos do envelhecimento e da saúde na velhice podem subsidiar ações favoráveis à melhoria da qualidade de vida $\mathrm{e}$ à promoção do envelhecimento saudável (ASSUMPÇÃO et al., 2018).

\section{Conclusões}

A avaliação do estado nutricional é de suma importância para a população idosa, pois torna possível a realização da intervenção nutricional primária, auxiliando e melhorando a qualidade de vida desses indivíduos.

$\mathrm{O}$ presente estudo evidenciou que a maioria dos idosos apresentaram excesso de peso quando avaliados pelo IMC e risco de desnutrição quando avaliados pela MNA. Além disso, nas idades mais avançadas verificou-se que a prevalência de excesso de peso diminuiu quando avaliado através do IMC e o risco para desnutrição aumentou quando avaliado através da MNA.

O IMC faz parte da MNA, sendo esta um instrumento de rastreio específico para a população idosa e mais indicado para se detectar o risco nutricional, pois leva em consideração outros fatores, entre eles a ingestão alimentar.

Desta forma, sugere-se a realização de mais estudos comparativos, em outras amostras da população idosa, utilizando diferentes métodos de avaliação nutricional, no intuito de refletir sobre os resultados obtidos. Salienta-se, que este estudo apresenta limitações no que tange ao tamanho amostral e por ter sido realizado apenas uma avaliação antropométrica.

Evaluation of nutritional status of elderly using the body mass index and the mini nutritional assessment

\section{Abstract}

The elderly may present changes in nutritional status. The most important changes in body composition are the increase in total body fat and the reduction of muscle tissue, which contribute to the occurrence of nutritional disorders, such as malnutrition or excess weight. This study to evaluate the nutritional status of elderly people attending a coexistence group in the municipality of Boa Vista das Missões/RS. Participants were 16 elderly people, and the majority $(81.3 \%)$ were women and were between 61 and 79 years old. The data were collected was based on the body mass index and the mini nutritional assessment, in addition to a questionnaire with closed questions to characterize the sample. As for marital sta- 
tus, the majority $(62.5 \%)$ were married or living with partners and $87.5 \%$ lived with their relatives. Through body mass index, it was verified that $56.3 \%$ were overweight. The results of mini nutritional assessment showed that $50 \%$ presented a risk of malnutrition. In conclusion, most of the elderly were overweight when evaluated by BMI and risk of malnutrition when evaluated by mini nutritional assessment. In addition, in the more advanced ages it was verified that the prevalence of overweight decreased when evaluated through the body mass index and the risk for malnutrition increased when evaluated through the mini nutritional assessment. Both methods are valid and widely used in nutritional assessment of the elderly.

Keywords: Aging. Body mass index. Mini nutritional assessment.

\section{Referências}

ALENCAR, M. G.; LEITÃO, M. B. S.; PRADO, L. V. S. Evolução do estado nutricional de pacientes internados na clínica médica de um hospital filantrópico de Pernambuco - Brasil. Nutrición Clínica y Dietética Hospitalaria, v. 35 , n. 3, p. 8-16, 2015.

ALMEIDA, M. F. et al. Anthropometric changes in the Brazilian cohort of older adults: SABE Survey (Health, Well-Being, and Aging). Journal of Obesity, [s.v], [s.n.], p. 1-9, Abr. 2013. DOI: http://dx.doi. org/10.1155/2013/695496

ASSUMPÇÃO, D. et al. Fatores associados ao baixo peso em idosos comunitários de sete cidades brasileiras: Estudo FIBRA. Ciência \& Saúde Coletiva, Rio de Janeiro, v. 23, n. 4, p. 1143-1150, abr. 2018.

AZEVEDO, A. S. et al. Perfil nutricional de pacientes adultos e idosos hospitalizados. Saúde e Pesquisa, Maringá, v. 9, n. 1, p. 25-29, 2016.
BEFORT, C. A.; NAZIR, N.; PERRI, M. G. Prevalence of obesity among adults from rural and urban areas of the united states: Findings from NHANES (2005-2008). Journal of Rural Health, v. 28, n. 4, p. 392-397, 2012.

BRASIL. Instituto Brasileiro de Geografia e Estatística (IBGE). Censo demográfico 2010: características gerais da população, religião e pessoas com deficiência. Rio de Janeiro: IBGE, 2010.

BRASIL. Instituto Brasileiro de Geografia e Estatística (IBGE). Expectativa de vida do brasileiro sobe para 75,8 anos. Rio de Janeiro: IBGE, 2017.

CARDOZO, N. R. et al. Estado nutricional de idosos atendidos por unidades de saúde da família na cidade de Pelotas-RS. Braspen Journal, São Paulo, v. 32, n. 1, p. 94-98, 2017.

CAVALCANTE, L. S.; COUTINHO, P. T. Q.; BURGOS, M. G. P. A. Aplicabilidade da MAN - mini avaliação nutricional em idosos diabéticos. Nutrición Clínica y Dietética Hospitalaria, v. 37, n. 1, p. 67-74, 2017.

CORTEZ, A. C. L.; MARTINS, M. C. C. Indicadores antropométricos do estado nutricional em idosos: uma revisão sistemática. UNOPAR Científica Ciências Biológicas e da Saúde, Londrina, v. 14, n. 4, p. 271-277, 2012.

CUPPARI, L. et al. Avaliação nutricional. In: CUPPARI, L. Guia de nutrição: nutrição clínica no adulto. 2a ed. Barueri: Manole, 2005. p. 89-127.

GARCIA, C. A. M. S.; MORETTO, M. C.; GUARIENTO, M. E. Estado nutricional e qualidade de vida em idosos. Revista $d a$ Sociedade Brasileira de Clínica Médica, xx, v. 14, n. 1, p. 52-56, jan./mar. 2016.

GUEDES, A. B.; GAMA, C. R.; TIUSSI, A. R. Avaliação nutricional subjetiva do idoso: Avaliação Subjetiva Global (ASG) versus Mini Avaliação Nutricional (MAN®). Comunicação em Ciências da Saúde, Brasília, v. 19, n. 4, p. 375-384, out./dez. 2008. 
GUIGOZ, Y.; VELLAS, B.; GARRY, P. J. Mini Nutritional Assessment: a practical assessment tool for grading the nutritional state of elderly patients. Facts and Research in Gerontology, v. 4, n. 2, p. 15-59, 1994.

HABIB, S. S. Body mass index and body fat percentage in assessment of obesity prevalence in Saudi adults. Biomedical and Environmental Sciences, v. 26, n. 2, p. 94-99, Feb. 2013.

HERNÁNDEZ-LUIS, R. et al. Prognostic value of physical function tests and muscle mass in elderly hospitalized patients. A prospective observational study. Geriatrics $\&$ Gerontology International, v. 18, n. 1, p. 57-64, 2018.

INZITARI, M. et al. International Association Of Gerontology And Geriatrics (IAGG) task force for nutrition in the elderly. Nutrition in the age-related disablement process. Journal of Nutrition, Health \& Aging, v. 15, n. 8, p. 599-604, Ago. 2011.

LIPSCHITZ, D. A. Screening for nutritional status in the elderly. Primary Care, v. 21, n. 1, p. 55-67, Mar. 1994.

MANTOVANI, L. M.; VIEBIG, R. F.; MORIMOTO, J. M. Associação entre estado nutricional e vulnerabilidade em idosos institucionalizados, Braspen Journal, São Paulo, v. 33, n. 2, p. 181-7, 2018.

MARTINS, M. V. et al. Consumo alimentar de idosos e sua associação com o estado nutricional. HU Revista, Juiz de Fora, v. 42, n. 2, p. 125-131, jun./ago. 2016.

MENEZES, T. N. et al. Perfil antropométrico dos idosos residentes em Campina Grande - PB. Revista Brasileira de Geriatria e Gerontologia, Rio de Janeiro, v. 16, n. 1, p. 19-27, 2013.

MONTEIRO, M. A. M. Percepção sensorial dos alimentos em idosos. Revista Espaço para Saúde, Londrina, v. 10, n. 2, p. 34-42, jun. 2009.
MUSSOI, T. D. Avaliação nutricional na prática clínica - da gestação ao envelhecimento. Rio de Janeiro: Guanabara Koogan; 2014.

ONGAN, D.; RAKICIOGLU, N. Nutritional status and dietary intake of institutionalized elderly in Turkey: A cross-sectional, multi-center, country representative study. Archives of Gerontology and Geriatrics, v. 61, n. 2, p. 271-276, 2015.

PAZ, R. C.; FAZZIO, D. M. G.; SANTOS, A. L. B. Avaliação nutricional em idosos institucionalizados. Revista de Divulgação Científica Sena Aires, Valparaiso de Goiás, v. 1, n. 1, p. 9-18, jan./jun. 2012.

PEREIRA, H. E. F. et al. Perfil nutricional e dietético de idosos atendidos nas Estratégias de Saúde da Família do norte de Minas Gerais. Revista de Atenção Primária à Saúde, Juiz de Fora, v. 21, n. 2, p. 259-266, abr./ jun. 2018.

PEREIRA, I. F. S.; SPYRIDES, M. H. C.; ANDRADE, L. M. B. Estado nutricional de idosos no Brasil: uma abordagem multinível. Cadernos de Saúde Pública, Rio de Janeiro, v. 32, n. 5, p. 1-12, 2016.

PEREIRA, M. L. A. S. et al. Nutritional status of institutionalized elderly Brazilians: a study with the Mini Nutritional Assessment. Nutricion Hospitalaria, v. 31, n. 3, p. 11981204, 2015.

PEREZ-FLORES, J. E. et al. Evaluación del estado nutricional al ingreso hospitalario y su asociación con la morbilidad y mortalidad en pacientes mexicanos. Nutricion Hospitalaria, v. 33, n. 4, p. 872-878, 2016.

ROCHA, É. C. P. F. et al. Perfil nutricional de idosas residentes em instituição de longa permanência da cidade do Recife/PE a partir de diferentes métodos antropométricos. Nutrición Clínica y Dietética Hospitalaria, v. 36, n. 2, p. 38-44, 2016.

SALMASO, F. V. et al. Análise de idosos ambulatoriais quanto ao estado nutricional, sarcopenia, função renal e densidade óssea. Arquivos Brasileiros de Endocrinologia \& 
Metabologia, São Paulo, v. 58, n. 3, p. 226231, 2014.

SANCHEZ-RODRIGUEZ, D. et al. Malnutrition in postacute geriatric care: Basic ES$\mathrm{PEN}$ diagnosis and etiology based diagnoses analyzed by length of stay, in-hospital mortality, and functional rehabilitation indexes. Archives of Gerontology and Geriatrics, v. 73, p. 169-176, 2017.

SANTANA, D. C. et al. Avaliação nutricional de idosos institucionalizados na Zona Sul de São Paulo. Revista Kairós Gerontologia, São Paulo, v. 19, n. 22, p. 403-416, 2016.

SANTOS, A. C. O.; MACHADO, M. M. O.; LEITE, E. M. Envelhecimento e alterações do estado nutricional. Revista Brasileira de Geriatria \& Gerontologia, Rio de Janeiro, v. 4, n. 3, p. 168-175, 2010.

SANTOS, A. L. et al. Avaliação do perfil sociodemográfico e nutricional na diferença entre homens e mulheres idosos ingressantes no programa Universidade Aberta para a Maturidade. Revista de enfermagem UFPE online, Recife, v. 11, n. 1, p. 327-333, 2017.

SCHERER, R. et al. Estado nutricional e prevalência de doenças crônicas em idosos de um município do interior do Rio Grande do Sul. Revista Brasileira de Geriatria e Gerontologia, Rio de Janeiro, v. 16, n. 4, p. 769-779, out./dez. 2013.

SILVA, J. L. et al. Fatores associados à desnutrição em idosos institucionalizados. Revista Brasileira de Geriatria e Gerontologia, Rio de Janeiro, v. 18, n. 2, p. 443-451, 2015.

SOUZA, K. T. et al. Baixo peso e dependência funcional em idosos institucionalizados de Uberlândia - MG, Brasil. Ciência \& Saúde Coletiva, Rio de Janeiro, v. 19, n. 8, p. 35133520, 2014.

SOUZA, R. et al. Avaliação antropométrica em idosos: estimativas de peso e altura e concordância entre classificações de IMC. Revista Brasileira de Geriatria e Gerontologia, Rio de Janeiro, v. 16, n. 1, p. 81-90, jan./ mar. 2013.
SPEROTTO, F. M.; SPINELLI, R. B. Avaliação nutricional em idosos independentes de uma instituição de longa permanência no município de Erechim - RS. Revista Perspectiva, Erechim, v. 34, n. 125, p. 105-116, mar. 2010.

TOMASI, E. et al. Utilização de serviços de saúde no Brasil: associação com indicadores de excesso de peso e gordura abdominal. Cadernos de Saúde Pública, Rio de Janeiro, v. 30, n. 7, p. 1515-1524, 2014.

VOLPINI, M. M.; FRANGELLA, V. S. Avaliação nutricional de idosos institucionalizados. Einstein, São Paulo, v. 11, n. 1, p. 32-40, 2013.

ZATTAR, L. C. et al. Prevalência e fatores associados à pressão arterial elevada, seu conhecimento e tratamento em idosos no sul do Brasil. Cadernos de Saúde Pública, Rio de Janeiro, v. 29, n. 3, p. 507-521, 2013. 